\title{
Structure and electronic properties of graphyne layers modeled on layers of graphene $\mathrm{L}_{3-12}$
}

\author{
V. V. Mavrinskii ${ }^{1}$, E. A. Belenkov ${ }^{2, \dagger}$ \\ †belenkov@csu.ru \\ ${ }^{1}$ Nosov Magnitogorsk State Technical University, 38 Lenin st., Magnitogorsk, 455000, Russia \\ ${ }^{2}$ Chelyabinsk State University, 129 Bratiev Kashirinykh st., Chelyabinsk, 454001, Russia
}

In the present paper, a theoretical study of the structure and electronic properties of new polymorphic conformations of graphyne layers modeled on layers of graphene $\mathrm{L}_{3-12}$ was performed. Graphyne layers have been constructed by replacing carbon-carbon bonds between three-coordinated ( $\mathrm{sp}^{2}$-hybridized) atoms in the graphene layer of $\mathrm{L}_{3-12}$ with diatomic carbyne chains. Geometric optimization and examination of electronic properties of novel graphyne architectures were performed within the framework of density functional theory using the gradient approximation. Calculations have shown the possibility of stable existence of three main polymorphic conformations of graphyne layers. The graphyne layers were designed by incorporating a carbyne chain into the initial $\mathrm{L}_{3-12}$-graphene layer by following rules: for the $\gamma-\mathrm{L}_{3-12}$-graphyne layer, one bond of each three-coordinated atom was substituted by a carbyne chain, for the $\beta-\mathrm{L}_{3-12}$-graphyne layer, two bonds of those atoms were replaced, and for $\alpha-\mathrm{L}_{3-12}$-graphyne layers three bonds were replaced. The sublimation energy of the graphyne layers is in the range from 6.52 to $6.61 \mathrm{eV} /$ atom that is less than the sublimation energy of the original $\mathrm{L}_{3-12}$ graphene layer $(6.66 \mathrm{eV} /$ atom) as well as the sublimation energy of hexagonal graphene $(7.76 \mathrm{eV} /$ atom). However, the value of the sublimation energy of the graphyne layers is in the range of experimentally synthesized carbon materials that are stable under normal conditions. All the graphyne layers studied in this work are semiconductors with the energy gaps widths from 0.18 to $0.91 \mathrm{eV}$.

Keywords: graphene, graphyne, polymorphism, modeling, crystal structure, density of electronic states.

УДК: 538.915

\section{Структура и электронные свойства графиновых слоев, сформированных на основе графена $\mathrm{L}_{3-12}$}

\author{
Мавринский В. В. ${ }^{1}$, Беленков Е.А. ${ }^{2, \dagger}$ \\ ${ }^{1}$ Магнитогорский государственный технический университет им. Г.И. Носова, \\ пр. Ленина 38, Магнитогорск, 455000, Россия \\ 2Челябинский государственный университет, ул. Братьев Кашириных, 129, Челябинск, 454001, Россия
}

В данной работе выполнено теоретическое исследование структуры и электронных свойств новых полиморфных разновидностей графиновых слоев, модельно построенных на основе слоев графена $\mathrm{L}_{3-12}$. Графиновые слои были построены в результате замены в графеновом слое $\mathrm{L}_{3-12}$ углерод-углеродных связей между трехкоординированными ( $\mathrm{sp}^{2}$-гибридизированными) атомами на двухатомные карбиновые цепочки. Геометрическая оптимизация модельно построенных структур и расчет их свойств были выполнены методом теории функционала плотности в градиентном приближении. В результате выполненных исследований установлена возможность устойчивого существования трех основных полиморофных разновидностей графиновых слоев. Слой $\gamma$ - $\mathrm{L}_{3-12}$-графина был получен заменой на карбиновую цепочку одной из связей у каждого трехкоординированного атома в исходном графеновом слое, слой $\beta-\mathrm{L}_{3-12}$-графина - заменой двух связей, а слой $\alpha-\mathrm{L}_{3-12}$-графина - заменой трех связей. Энергия сублимация графиновых слоев находится в диапазоне от 6.52 до 6.61 эВ/атом, что меньше энергии сублимации как исходного графенового слоя $\mathrm{L}_{3-12}(6.66$ эВ/атом), так и сублимационной энергии гексагонального графена (7.76 эB/атом). Однако значение энергии сублимации графиновых слоев находится в диапазоне экспериментально синтезированных углеродных материалов устойчиво существующих при нормальных условиях. Все графиновые слои, изученные в работе являются полупроводниками с ширинами запрещенных зон от 0.18 до 0.91 эВ.

Ключевые слова: графен, графин, полиморфизм, моделирование, кристаллическая структура, плотность электронных состояний. 


\section{1. Введение}

Углеродные атомы в соединениях могут находится в двух, трех или четырехкоординированных состояниях, соответствующих $\mathrm{sp}, \mathrm{sp}^{2}$ и $\mathrm{sp}^{3}$ гибридизации электронных орбиталей атомов. Гибридные углеродные материалы состоят из углеродных атомов в состояниях с различной гибридизацией. Свойства таких гибридных соединений могут варьироваться в широком диапазоне при изменении соотношения атомов в состояниях с различной гибридизацией электронных орбиталей. Существует четыре класса гибридных углеродных соединений $\left(\mathrm{sp}+\mathrm{sp}^{2}, \mathrm{sp}+\mathrm{sp}^{3}, \mathrm{sp}^{2}+\mathrm{sp}^{3}\right.$ и $\left.\mathrm{sp}+\mathrm{sp}^{2}+\mathrm{sp}^{3}\right)$. Слоевые структуры, состоящие из двух- и трехкоординированных углеродных атомов (sp- и $\mathrm{sp}^{2}$-гибридизация электронных орбиталей), так называемые графины (graphyne), теоретически исследуются уже несколько десятилетий [1-7]. Однако впервые экспериментально синтезировать одну из структурных разновидностей графина - графдиин (graphdiyne) удалось только в 2010 [8]. В ряде недавних теоретических исследований была предложена модельная схема построения всех возможных структурных разновидностей графина в результатате трансформации структуры исходных графеновых слоев $[9,10]$. Для ряда графиновых слоев построенных при помощи этой модельной схемы на основе слоев графена $\mathrm{L}_{6}$ и $\mathrm{L}_{4-12}$ был выполнен расчет оптимальной структуры и свойств [9-12]. Данная работа продолжает эти исследования - в ней выполнено исследование графиновых слоев модельно формируемых на основе $\mathrm{L}_{3-12}$ полиморфной разновидности графена.

\section{2. Методика расчетов}

В качестве исходного слоя для модельного построения графиновых слоев в работе был взят $\mathrm{L}_{3-12}$ графеновый слой (Рис. 1) [13,14]. В этом слое все атомы находятся в одинаковых структурных состояниях, и каждый атом связан с соседними тремя ковалентными связями, имеющие длины $\mathrm{R}_{1}, \mathrm{R}_{2}$ и $\mathrm{R}_{3}\left(\mathrm{R}_{1}=\mathrm{R}_{2}\right)$. Структуру исходных слоев графина модельно строили в результате замены углерод-углеродных связей на карбиновые цепочки.

Замены проводили так, что у каждого трехкоординированного атома первичного графенового слоя была заменена на двухатомную карбиновую цепочку одна, две или три связи. В результате были построены три графиновых слоя $\gamma, \beta$ и а разновидностей.

Исходные структуры графиновых слоев, построенные по модельной схеме, были подвергнуты геометрической оптимизации методами теории функционала плотности (density functional theory, DFT) [15] в градиентном приближении (generalized gradient approximations, GGA) [16]. Для расчетов использовались трехмерные структуры, в которых слои располагались стопками. Чтобы получить информацию о структуре и свойствах изолированных слоев и исключить влияние соседних слоев на структуру и свойства отдельного слоя расстояние между слоями в стопках принимали равным 10 А․ Для расчетов электронных свойств и геометрически оптимизированной структуры использовался программный пакет Quantum ESPRESSO [17]. Плотности электронных состояний рассчитывали, используя набор k-точек: $12 \times 12 \times 12$. Волновые функции раскладывались по усеченному базисному набору плоских волн. Размерность набора базисных функций была ограничена значением $\mathrm{E}_{\text {cutoff }}=1$ кэВ. Методики расчетов, использованные в работе, были хорошо апробированы ранее при исследовании различных углеродных материалов [18-23].

\section{3. Результаты и обсуждение}

В результате выполненных расчетов была найдена геометрически оптимизированная структура трех графиновых слоев ( $\left.\alpha-\mathrm{L}_{3-12}, \beta-\mathrm{L}_{3-12}, \gamma-\mathrm{L}_{3-12}\right)$ изображения которых приведены на Рис. 2. Таким образом, на основе графена $\mathrm{L}_{3-12}$ возможно построение всего трех основных структурных разновидностей графина, в то время

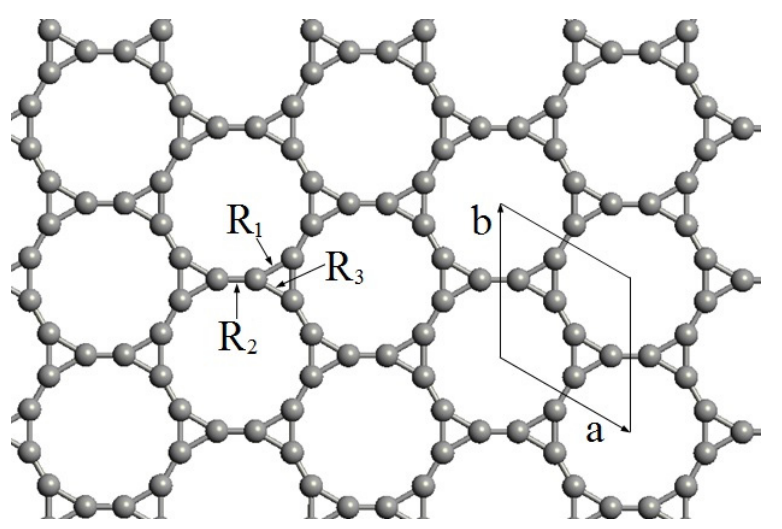

Рис. 1. Элементарная ячейка и кристаллическая структура исходного графенового слоя $\mathrm{L}_{3-12}$.

Fig. 1. Unit cell and crystal structure of the initial graphene layer $\mathrm{L}_{3-12}$.
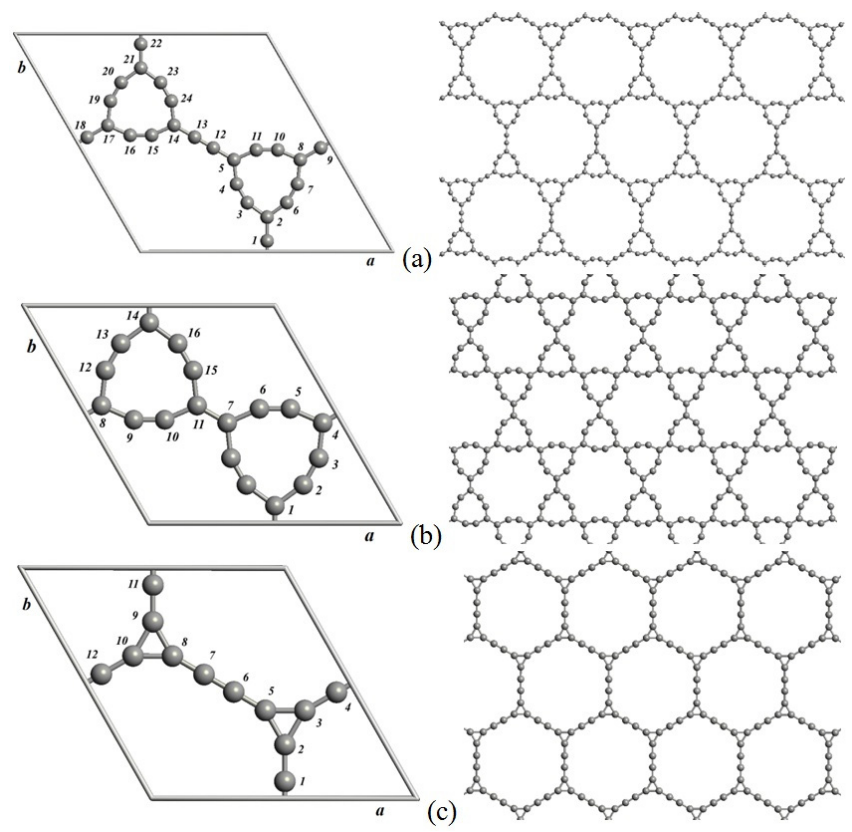

Рис. 2. Элементарные ячейки и кристаллические структуры графиновых слоев: $\alpha-\mathrm{L}_{3-12}$-графин (a); $\beta-\mathrm{L}_{3-12}$-графин (b); $\gamma$ - $\mathrm{L}_{3-12}$-графин (с).

Fig. 2. Unit cell and crystal structure of graphyne layers: $\alpha-\mathrm{L}_{3-12}$-graphyne (a); $\beta-\mathrm{L}_{3-12}$-graphyne (b); $\gamma-\mathrm{L}_{3-12}$-graphyne (c). 
как на основе слоев $\mathrm{L}_{6}, \mathrm{~L}_{4-8}$ и $\mathrm{L}_{4-6-12}$ могут быть получены по 7 основных структурных разновидностей - по одной $\alpha$ типа и по три для $\beta$ и $\gamma$ структур [10 - 12]. Невозможность существования ряда разновидностей $\beta$ и $\gamma$ графиновых слоев, обусловлена в данном случае особенностями структуры исходного графенового слоя $\mathrm{L}_{3-12}$.

После оптимизации карбиновые цепочки в графиновом слое $\gamma$ - $\mathrm{L}_{3-12}$ остаются прямыми (Рис. 2c), в слое $\beta-\mathrm{L}_{3-12}$-графина все карбиновые цепочки изогнутые (Рис. 2b), а в слое $\alpha-\mathrm{L}_{3-12}$-графина часть цепочек прямая и часть изогнутая (Рис. 2a). Численные значения структурных параметров графиновых слоев, а также графеновых слоев $\mathrm{L}_{6}$ и $\mathrm{L}_{3-12}$, приведены в Табл. 1. Кристаллические решетки всех изученных слоев графина относятся гексагональным разновидностям двухмерных решеток Браве. В элементарных ячейках графиновых слоев содержится от 12 до 24 атомов.
В графиновых слоях $\beta-\mathrm{L}_{3-12}$ и $\gamma-\mathrm{L}_{3-12}$ атомы находятся в двух кристаллографически эквивалентных состояниях, соответствующих двух- и трехкоординированным ( $\mathrm{sp}$ и $\mathrm{sp}^{2}$ гибридизироаанным) атомам. В структуре слоя $\alpha-\mathrm{L}_{3-12}$ три различных структурных состояния атомов. В результате этого межатомные связи в слоях $\beta-\mathrm{L}_{3-12}$ и $\gamma-\mathrm{L}_{3-12}$ бывают трех различных длин (Табл. 2) - это связи между sp-sp, sp-sp $\mathrm{p}^{2}$ и $\mathrm{sp}^{2}-\mathrm{sp}^{2}$ гибридизированными атомами. В слое $\alpha-\mathrm{L}_{3-12}$-графина имеются межатомные связи двух типов sp-sp, sp-sp ${ }^{2}$, но связи каждого из типов бывают двух различных длин (Табл. 2). Причиной различий в длинах углерод-углеродных связей является различия в их порядках [18], то есть в числе пар электронов локализованных в межатомных промежутках.

Выполненные расчеты порядков связей в графиновых слоях показали, что они не принимают целых значений что свидетельствует о делокализации $\pi$ электронов.

Табл. 1. Структурные параметры и свойства графиновых и графеновых слоев.

Table 1. Structural parameters and properties of graphyne and graphene layers.

\begin{tabular}{|c|c|c|c|c|c|}
\hline $\begin{array}{l}\text { Слой } \\
\text { Layer }\end{array}$ & $\alpha-L_{3-12}$ & $\beta-\mathrm{L}_{3-12}$ & $\gamma-\mathrm{L}_{3-12}$ & $\mathrm{~L}_{6}$ & $\mathrm{~L}_{3-12}$ \\
\hline $\mathrm{a}, \AA$ & \multirow{2}{*}{14.688} & \multirow{2}{*}{10.156} & \multirow{2}{*}{9.759} & \multirow{2}{*}{2.491} & \multirow{2}{*}{5.130} \\
\hline $\mathrm{b}, \AA$ & & & & & \\
\hline$\gamma,{ }^{\circ}$ & 120 & 120 & 120 & 120 & 120 \\
\hline $\begin{array}{l}\mathrm{E}_{\text {total }},{ } \mathrm{B} / \mathrm{a} \text { атом } \\
\mathrm{E}_{\text {total }}, \mathrm{eV} / \text { atom }\end{array}$ & -156.11 & -156.34 & -156.08 & -157.32 & -156.22 \\
\hline $\begin{array}{l}\Delta \mathrm{E}_{\text {total }},{ }^{\prime} \text { В/атом } \\
\Delta \mathrm{E}_{\text {total }}, \mathrm{eV} / \text { atom }\end{array}$ & 1.21 & 1.15 & 1.24 & 0 & 1.10 \\
\hline $\begin{array}{l}\mathrm{E}_{\text {sub }}, \ni \mathrm{B} / \text { aтом } \\
\mathrm{E}_{\text {sub }},{ } \mathrm{B} / \text { atom }\end{array}$ & 6.55 & 6.61 & 6.52 & 7.76 & 6.66 \\
\hline $\begin{array}{l}\Delta, \ni \mathrm{B} \\
\Delta, \mathrm{eV}\end{array}$ & 0.39 & 0.18 & 0.91 & 0.00 & 0 \\
\hline $\begin{array}{l}\rho, \mathrm{Mг} / \mathrm{M}^{2} \\
\rho, \mathrm{mg} / \mathrm{m}^{2}\end{array}$ & 0.25 & 0.40 & 0.29 & 0.74 & 0.52 \\
\hline $\begin{array}{l}\mathrm{N} \text {, атом } \\
\mathrm{N} \text {, atom }\end{array}$ & 24 & 18 & 12 & 2 & 6 \\
\hline $\mathrm{sp}: \mathrm{sp}^{2}$ & 3 & 2 & 1 & 0 & 0 \\
\hline
\end{tabular}

Табл. 2. Длины связей и углы между связями в графиновых слоях.

Table 2. The lengths of bonds and the angles between bonds in the graphyne layers.

\begin{tabular}{|c|c|c|c|c|c|}
\hline \multicolumn{2}{|c|}{$\begin{array}{c}\alpha-\mathrm{L}_{3-12} \text {-графин } \\
\alpha-\mathrm{L}_{3-12} \text {-graphyne }\end{array}$} & \multicolumn{2}{|c|}{$\begin{array}{c}\beta-\mathrm{L}_{3-12} \text {-графин } \\
\beta-\mathrm{L}_{3-12} \text {-graphyne }\end{array}$} & \multicolumn{2}{|c|}{$\begin{array}{c}\gamma-\mathrm{L}_{3-12} \text {-графин } \\
\gamma-\mathrm{L}_{3-12} \text {-graphyne }\end{array}$} \\
\hline $\mathrm{L}_{1-2}, \AA$ & 1.3849 & $\mathrm{~L}_{1-2}, \AA$ & 1.4264 & $\mathrm{~L}_{1-2}, \AA$ & 1.3469 \\
\hline $\mathrm{L}_{2-3}, \AA$ & 1.4250 & $\mathrm{~L}_{2-3}, \AA$ & 1.2501 & $\mathrm{~L}_{2-3}, \AA$ & 1.4502 \\
\hline $\mathrm{L}_{3-4}, \AA$ & 1.2517 & $\mathrm{~L}_{7-11}, \AA$ & 1.4035 & $\mathrm{~L}_{6-7}, \AA$ & 1.2659 \\
\hline $\mathrm{L}_{12-13}, \AA$ & 1.2533 & - & - & - & - \\
\hline$\beta_{3-2-6^{\prime}}{ }^{\circ}$ & 107.6 & $\beta_{1-2-3}, \circ$ & 156.2 & $\beta_{1-2-3}, \circ$ & 150 \\
\hline$\beta_{1-2-3},{ }^{\circ}$ & 126.2 & $\beta_{6-7-11},{ }^{\circ}$ & 126.2 & $\beta_{6-7-11},{ }^{\circ}$ & 60 \\
\hline$\beta_{2-3-4}, \circ$ & 156.2 & $\beta_{3-4-5}, \circ$ & 107.6 & $\beta_{3-4-5}, \circ$ & 180 \\
\hline$\beta_{5-12-13}, \circ$ & 180.0 & - & - & - & - \\
\hline $\operatorname{Def}\left(\mathrm{sp}^{2}\right),{ }^{\circ}$ & 24.8 & $\operatorname{Def}\left(\mathrm{sp}^{2}\right),{ }^{\circ}$ & 24.8 & $\operatorname{Def}\left(\mathrm{sp}^{2}\right){ }^{\circ}$ & 120 \\
\hline $\operatorname{Def}(\mathrm{sp})_{1},{ }^{\circ}$ & 0 & $\operatorname{Def}(\mathrm{sp}),^{\circ}$ & 47.8 & $\operatorname{Def}(\mathrm{sp}){ }^{\circ}$ & 0 \\
\hline $\operatorname{Def}(\mathrm{sp})_{2},{ }^{\circ}$ & 47.8 & - & - & - & - \\
\hline
\end{tabular}


Полная энергия взаимодействия атомных ядер и электронов, приходящаяся на один атом в графиновых слоях $\left(\mathrm{E}_{\text {total }}\right)$ изменяется в диапазоне от -156.34 до -156.08 эВ/атом, что выше соответствующего значения для гексагонального графена -157.32 эВ/атом и для $\mathrm{L}_{3-12}$ графена -156.22 эВ/атом (Табл. 1). Разница полных энергий $\left(\Delta \mathrm{E}_{\text {total }}\right)$ по сравнению со слоем $\mathrm{L}_{6}$ составляет от 1.15 до 1.24 эВ/атом. Энергия сублимации была найдена как разница между полной энергией отдельного атома в графиновом или графеновом слое и энергией изолированного углеродного атома (расчетное значение этой энергии -149.56 эВ/атом). Значения энергий сублимации графиновых слоев на $14-15 \%$ меньше соответствующего значения для слоя гексагонального графена (Табл. 1). Максимальная энергия сублимации наблюдается для $\beta-\mathrm{L}_{3-12}$-графина, минимальная для $\gamma-\mathrm{L}_{3-12}$-графина.

Результаты расчетов плотности электронных состояний для трех графиновых слоев приведены на Рис. 3. Численные значения ширин запрещенных зон на уровне энергии Ферми приведены в Табл. 1. Ширины запрещенных зон $\Delta$ графиновых слоев оказались в диапазоне от 0.18 до 0.91 эВ. Это свидетельствует о том, что эти графиновые слои должны проявлять полупроводниковые свойства. Плотность слоев графина $\rho$ оказалась меньше плотности графенового слоя $\mathrm{L}_{6}$ : меньше всего (в 1,85 раза) отличается плотность $\beta$-графина, наибольшие отличия (в 2,96 раза) наблюдаются для $\alpha$-графина.

\section{4. Заключение}

Таким образом, в работе были выполнены DFT-GGA расчеты электронных свойств и кристаллической структуры трех основных структурных разновидностей графина ( $\alpha-\mathrm{L}_{3-12}, \beta-\mathrm{L}_{3-12}$ и $\left.\gamma-\mathrm{L}_{3-12}\right)$, получающихся из графенового слоя $\mathrm{L}_{3-12}$. Эти графиновые соединения являются разновидностью новых гибридных углеродных материалов, состоящих из $\mathrm{sp}+\mathrm{sp}^{2}$ гибридизированных углеродных атомов. Отсутствие ряда других структурных типов, характерных для слоев графина сформированных на основе графеновых политипов $\mathrm{L}_{6}, \mathrm{~L}_{4-8}$ и $\mathrm{L}_{4-6}$, обусловлена особенностями структуры исходного графенового слоя $\mathrm{L}_{3-12}$. Рассчитанные значения энергий сублимации графиновых слоев оказались в диапазоне энергий сублимации экспериментально синтезированных углеродных соединений и, вероятно, они могут устойчиво существовать при нормальных условиях. Невысокие значения энергий сублимации графиновых слоев по сравнению со значением аналогичных характеристик для графеновых слоев, обусловлены значительной деформацией углов между связями, характеризуемой деформационными параметрами Def (Табл. 2). Расчеты зонной структуры и плотности электронных состояний графиновых соединений указывают на то, что изученные слои $\alpha-\mathrm{L}_{3-12}, \beta-\mathrm{L}_{3-12}$, и $\gamma-\mathrm{L}_{3-12}$ должны быть узкозонными полупроводниками.
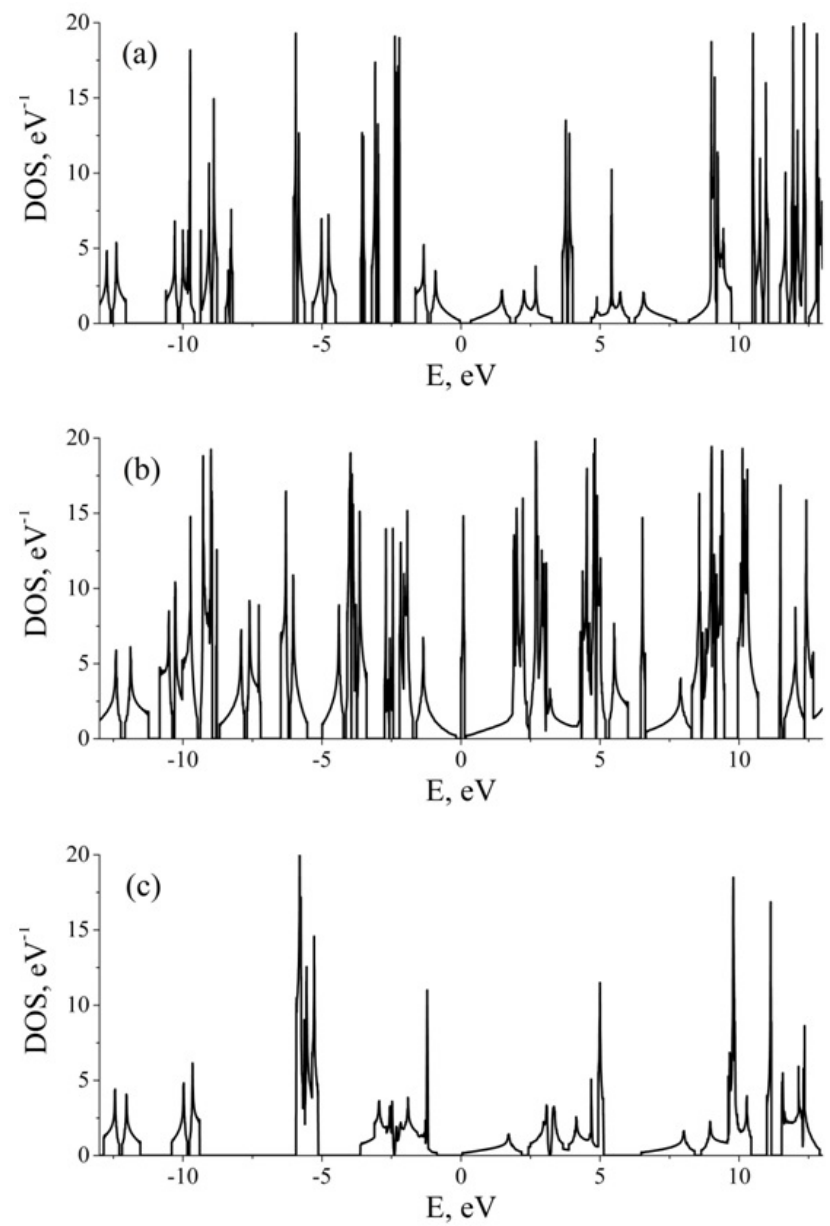

Рис. 3. Плотность электронных состояний графиновых слоев: $\alpha-\mathrm{L}_{3-12}$-графин (a); $\beta-\mathrm{L}_{3-12}$-графин (b); $\gamma-\mathrm{L}_{3-12}$-графин (c).

Fig. 3. Electronic density of states graphyne layers: $\alpha-\mathrm{L}_{3-12}$-graphyne (a); $\beta-\mathrm{L}_{3-12}$-graphyne (b); $\gamma$ - $\mathrm{L}_{3-12}$-graphyne (c).

Благодарность/Acknowledgements. Авторы благодарят Фонд перспективных научных исследований ЧелГУ за финансовую поддержку проведенного исследования./This work was supported by Foundation for perspective scientific research of Chelyabinsk State University.

\section{Литература/References}

1. R. H. Baughman, H. Eckhardt, M. Kertesz. J. Chem. Phys. 87, 6687 (1987). DOI: 10.1063/1.453405

2. N. Narita, S. Nagai, S. Suzuki, K. Nakao. Phys. Rev. B. 58, 11009 (1998). DOI: 10.1103/PhysRevB.58.11009

3. E.A. Belenkov, I.V. Shakhova. Physics of the Solid State. 53(11), 2385 (2011). (in Russian) [Е.А.Беленков, И.В. Шахова. Физика твердого тела. 53(11), 2265 (2011).] DOI: 10.1134/S1063783411110059

4. B. G. Kim, H. J. Choi. Phys. Rev. B. 86, 115435 (2012). DOI: 10.1103/PhysRevB.86.115435

5. C.-W. Kim, S.-H. Kang, and Y.-K. Kwon. Phys. Rev. B. 92, 
245434 (2015). DOI: 10.1103/PhysRevB.92.245434

6. P. Zhang, Q. Song, J. Zhuang, X.-J. Ning. Chem. Phys. Let. 689, 185 (2017). DOI: 10.1016/j.cplett.2017.10.026

7. J. Qu, H. Zhang, J. Li, S. Zhao, T. Chang. Carbon. 120, 350 (2017). DOI: 10.1016/j.carbon.2017.05.051

8. G. Li, Y. Li, H. Liu, et al. Chem. Commun. 46, 3256 (2010). DOI: 10.1039/B922733D

9. В.В. Мавринский, Т.Е. Беленкова, В.М. Чернов, Е.А. Беленков. Вестник Челябинского государственного университета. 316(23), 31 (2013).

10. E.A. Belenkov, V.V. Mavrinskii, T.E. Belenkova, V. M. Chernov. J.Exp. Theor. Phys. 120(5), 820 (2015). (in Russian) [Е.А. Беленков, В.В. Мавринский, Т.Е. Беленкова, В. М. Чернов. ЖЭТФ. 120(5), 949 (2015).] DOI: 10.1134/S1063776115040214

11. Т.Е. Беленкова, В. М. Чернов. Фундаментальные проблемы современного материаловедения. 13(2), 149 (2016).

12. T. Belenkova, V. Chernov, V. Mavrinskii. Materials Science Forum. 845, 239 (2016). DOI: 10.4028/www.scientific.net/MSF.845.239

13. E. A. Belenkov, V. A. Greshnyakov. New Carbon Materials. 28(4), 273 (2013). DOI: 10.1016/S1872-5805(13)60081-5

14. E.A. Belenkov, A.E. Kochengin. Physics of the Solid State. 57(10), 2126 (2015). (in Russian) [Е.А. Беленков, А.Е. Коченгин. Физика твердого тела. 57(10), 2071 (2015).] DOI: 10.1134/S1063783415100030

15. W. A. Koch, M. C. Holthausen. Chemist's guide to density functional theory. $2^{\text {nd }}$ edition. Weinheim, Wiley-VCH Verlag GmbH (2001) 293 p.

16. J. P. Perdew, J. A. Chevary, S. H. Vosko et al. Phys. Rev. B. 46, 6671 (1992). DOI: 10.1103/PhysRevB.46.6671

17. P. Giannozzi, S. Baroni, N. Bonini et al. J. Phys. Condens. Matter. 21, 395502 (2009).
DOI: $10.1088 / 0953-8984 / 21 / 39 / 395502$

18. E. A. Belenkov. Inorganic Materials. 37(9), 928 (2001). (in Russian) [Е. А. Беленков. Неорганические материалы. 37(9), 1094 (2001).] DOI 10.1023/A:101160191560018

19. D.S. Lisovenko, J.A. Baimova, L.Kh. Rysaeva, V.A. Gorodtsov, A.I. Rudskoy, S.V. Dmitriev. Phys. Status Solidi B. 253(7), 1295 (2016). DOI: $10.1002 /$ pssb.201600049

20. Н.В. Новиков, М.М. Маслов, К.П. Катин, В. С. Прудковский. Письма о материалах. 7(4), 433 (2017). DOI: 10.22226/2410-3535-2017-4-433-436

21. I.P. Lobzenko, G.M. Chechin, G.S. Bezuglova, Y. A. Baimova, E. A. Korznikova, S. V. Dmitriev. Physics of the Solid State. 58(3), 633 (2016). (in Russian) [И.П. Лобзенко, Г.М. Чечин, Г.С. Безуглова, Ю.А. Баимова, Е.А. Корзникова, С.В. Дмитриев. Физика твердого тела. 58(3), 616 (2016).] DOI: $10.1134 /$ S1063783416030203

22. Э.И. Беленкова. В книге: Новые технологии в материаловедении. Материалы I научно-практической конференции с международным участием. Уфа, БашГУ (2015) 308 с. Моделирование структурной трансформации углеродных материалов, обусловленное изменением размеров нанокристаллитов. c. $176-177$.

23. A.V. Savin, E.A. Korznikova, I.P. Lobzenko, Yu.A. Baimova, S. V. Dmitriev. Physics of the Solid State. 58(6), 1278 (2016). (in Russian) [А. В. Савин, Е.А. Корзникова, И.П. Лобзенко, Ю.А. Баимова, С.В. Дмитриев. Физика твердого тела. 58(6), 1236 (2016).] DOI: 10.1134/S1063783416060317

24. K.S. Grishakov, K.P. Katin, M.M. Maslov. Advances in Physical Chemistry. 2016, 1862959 (2016). DOI: $10.1155 / 2016 / 1862959$ 